\title{
Dislodgement of a magnesium bioresorbable scaffold from the delivery system in the left main: A true "nightmaris"
}

\author{
Carlos Cortés ${ }^{1,2^{*}}$, Milosz Jaguszewski1 ${ }^{1,34^{*}}$, Michele Schincariol ${ }^{3}$, \\ María-Alexandra Durán-Cortés ${ }^{1}$, Ula Limon ${ }^{1}$, Juan Luis Gutiérrez-Chico ${ }^{1,3}$ \\ ${ }^{1}$ Interventional Cardiology, Klinikum Frankfurt (Oder), Germany \\ ${ }^{2}$ Interventional Cardiology Department, Hospital Clínico Universitario de Valladolid, ICICOR, Valladolid, Spain \\ ${ }^{3}$ Institute of Cardiovascular Translational Research of Atlantic (ICTRA), Berlin, Germany \\ ${ }^{4}$ First Department of Cardiology, Medical University of Gdansk, Poland
}

This paper was guest edited by Prof. Sang-Wook Kim

A 69-year-old male patient with hypertension and hypercholesterolemia underwent percutaneous coronary intervention (PCI) of the left anterior descending artery (LAD), from mid to ostial, with implantation of three overlapping everolimuseluting stents (EES). PCI of a lesion in the distal left circumflex (LCx) (Supplementary Video 1 - see journal website) was scheduled 6 months later. Then an optical coherence tomography (OCT) pullback pre-PCI showed a predominantly fibrotic plaque of $19 \mathrm{~mm}$ length in the distal LCx. Predilation 1:1 with a scoring-balloon (Angiosculpt ${ }^{\mathrm{TM}} 3.5 \times 15 \mathrm{~mm}$, Biotronik, Berlin, Germany) was performed. A magnesium bioresorbable scaffold (Magmaris ${ }^{\mathrm{TM}}$ $3.5 \times 20 \mathrm{~mm}$, Biotronik, Berlin, Germany) was then advanced towards the lesion, but it got unexpectedly stuck at the distal left main (LM), unable to move forward. The attempts to retrieve the device back into the guiding catheter resulted in dislodgement from the delivery system. OCT showed an angiographically inadvertent protrusion of the previously implanted EES into the LM (Fig. 1, red arrowheads), in which's mess the Magmaris was entangled (Fig. 1, white arrowheads; Supplementary Video 2 see journal website). Some struts were visible in the guiding-catheter (Fig. 1F). Three-dimensional OCT image fusion depicted clearly the pattern of the crimped Magmaris (Fig. 2.A, B), even though the automatic detection software failed to recognise the struts. The scaffold retrieval by means of a snare
(Amplatz Gooseneck microsnare ${ }^{\mathrm{TM}}, 4 \mathrm{~mm}$, Covidien, Plymouth, MN) was performed under stationary OCT guidance, due to its complete radiotransparency. The device was firmly snared, but it broke twice in small fragments when the operator tried to pull it back (Fig. 2C, arrowheads). In a third attempt, the scaffold was successfully snared in a slightly more distal position. In this occasion the device did not break while pulling back, so it could be finally retrieved (Fig. 2C).

This case highlights the instrumental role of invasive imaging in critical problem solving, becoming even more prominent in radiotransparent scaffolds [1]. It also points out the importance of ensuring a clear way from the guiding catheter up to the lesion: neither the previous OCT, focused on assessing the lesion, nor predilation manoeuvres noticed the protrusion into the LM of a previously implanted EES. A careful assessment of the whole navigation pathway with invasive imaging might be advisable if PCIs have been previously performed in adjacent segments.

Conflict of interest: None declared

\section{Reference}

1. Jaguszewski MJ, Cortés C, Gutiérrez-Chico JL. Implantation of magnesium-bioresorbable scaffolds in a bifurcation under optical coherence tomography guidance. Eur Heart J. 2017; 38(25): 2017-2018, doi: 10.1093/eurheartj/ehw539, indexed in Pubmed: 27941012 .

Address for correspondence: Prof. Juan Luis Gutiérrez-Chico, MD, PhD, FESC, FACC, Head of Interventional Cardiology, Klinikum Frankfurt (Oder), Müllroser Chaussée 7, 15236 - Frankfurt (Oder), Germany, tel: +49 (0) 17630585019 ,

+34 615 319370, e-mail: juanluis.gutierrezchico@ictra.es

Received: 10.05.2017 Accepted: 13.07.2017

*Equally contributed 


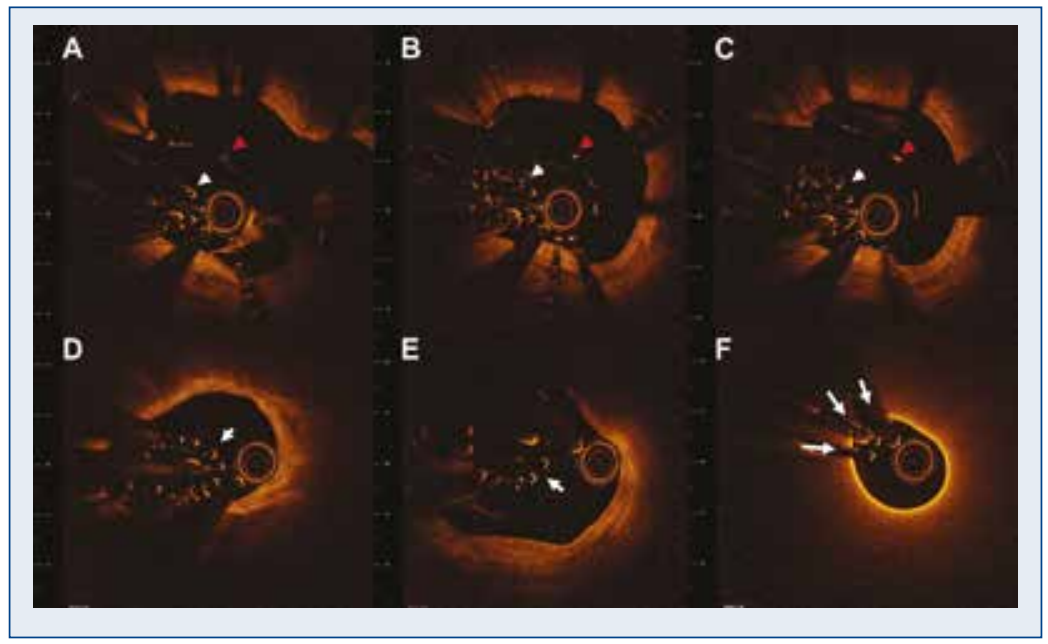

Figure 1A-F. Optical coherence tomography (OCT) after dislodgement of the magnesium bioresorbable scaffold (MgBRS) from its delivery system in the left main (LM). The crimped MgBRS (white arrowheads) is clearly visible, with a guidewire inside the scaffold lumen. A second guidewire outside the scaffold lumen supports the OCT catheter. A stent previously implanted in the left anterior descending artery is amply protruding into the LM (red arrowheads), so the MgBRS got entangled in its mesh (A-C). Struts of the MgBRS can be seen in the guiding catheter (F).

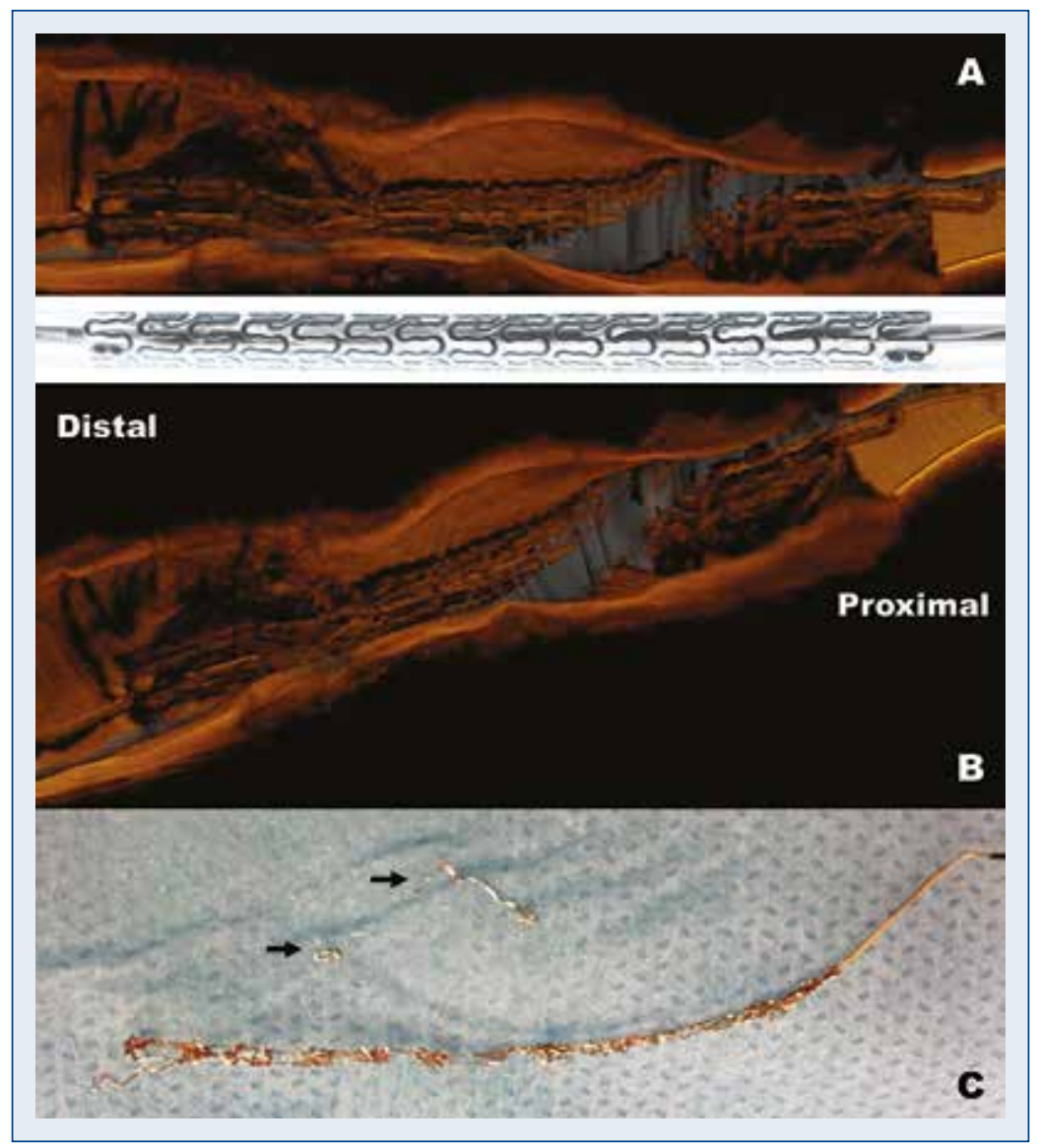

Figure 2. Optical coherence tomography image fusion, rendering a three-dimensional depiction of the left main (A, B). The platform pattern of the crimped Magmaris bioresorbable scaffold (MgBRS) can be clearly recognised, with its proximal struts overhanging the guiding-catheter (A, B). The MgBRS could be removed from the left main by means of a snare (C). The snared scaffold broke twice in small pieces (C, arrowheads) while pulled back, before being successfully retrieved. 